\title{
Nonlinear Control of Buildings Subjected to Earthquakes by Using Sliding Mode Controller (SMC)
}

\author{
Borzoo Afkham (Corresponding author) \\ MAPNA Electrical and Control Engineering \& Manufacturing Co. (MECO) \\ MAPNA Blvd, $6^{\text {th }} \mathrm{Km}$ of Malard Road, Fardis, Karaj 31176, Iran, P.O. Box 31585-1551 \\ Tel: 98-261-6638-0019Ｆax: 98-261-661-8020Ｅ-mail:afkham@mapnaec.com
}

Saeedreza Ehteram

MAPNA Electrical and Control Engineering \& Manufacturing Co. (MECO)

MAPNA Blvd, $6^{\text {th }} \mathrm{Km}$ of Malard Road, Fardis, Karaj 31176, Iran, P.O. Box 31585-1551

Tel: 98-261-6638-0019Ｆax: 98-261-661-8020Ｅ-mail:ehteram@mapnaec.com

\begin{abstract}
Earthquakes are responsible for the destruction and severe damage to buildings and structures because of their peak accelerations. The objective of this paper is to design controllers for buildings which are modeled using nonlinear models and subjected to earthquakes. The designed nonlinear controllers ought to be able to significantly reduce the floor displacements when a building is subjected to earthquakes. The nonlinear controllers are designed for buildings which are subject to earthquakes are based on the Sliding Mode Control (SMC) technique. The designed controllers are simulated using the Matlab/Simulink software. The simulation results indicate that the proposed controllers work well.
\end{abstract}

Keywords: Nonlinear control, Sliding Mode Controller (SMC), Earthquake

\section{Introduction}

An earthquake is the result of the abrupt release of energy of the earth's crust; it is among the most feared and annihilating natural disasters. Earthquakes strikes almost without prior notice; they last only few seconds and they can leave death and destruction. Earthquakes may result in disease, lack of basic necessities, loss of life, property damage, roads and bridges damage, the collapse of buildings or the destabilization of the base of buildings and structures, etc.

The application of linear control schemes to civil engineering structures has been investigated in recent years. However, the efficacy of these methodologies in the presence of near field external forces (such as earthquakes) is not very adequate. This is the case because linear controllers rely on the assumption of small range of operation of the system. However, when the required operation range is large, a linear controller is likely to perform poorly or to lead to instability.

A lot of the previous research works in the area of seismically excited building/structures have used linear models of the buildings and linear control methodologies. Generally, this is not very adequate because structures which are subject to near field earthquakes behave nonlinearly due to the excessive excitation levels and near field ground motions.

\section{Design of sliding mode controller}

\subsection{Summary of nonlinear building model}

The earthquake ground motion is characterized by high peak acceleration and velocity pulses with long period components as well as large ground displacements. Such characteristics are responsible for severe damages to flexible structures. The peak ground acceleration occurs in the form of shocks to buildings.

To assess the basic performances and examine the physical significance of the proposed nonlinear controllers, a three-story building structure equipped with nonlinear dampers and spring devices on each floor is analyzed when subjected to an earthquake excitation. The nonlinear dampers and spring devices are assumed to be installed between the floors as shown in Figure 1. Agrawal and Yang proposed nonlinear controllers for seismic excited inelastic buildings taking in consideration nonlinear dampers, springs and interaction between floors. Similar to the works of Agrawal and Yang, a 3-story base-isolated building subject to one dimensional earthquake ground acceleration is considered. 


\subsection{Nonlinear equations of building motion}

The equations of motion for 3-story base-isolated building subject to one dimensional earthquake ground acceleration are:

$$
\begin{aligned}
& m_{1}\left(\ddot{x}_{1}(t)-\ddot{x}_{o}(t)\right)+F_{c}\left[\dot{x}_{1}(t)\right]+F_{c}\left[\dot{x}_{2}(t)\right]+F_{S}\left[x_{1}(t)\right]+F_{S}\left[x_{2}(t)\right]=u_{A}(t) \\
& m_{2}\left(\ddot{x}_{2}(t)-\ddot{x}_{o}(t)\right)+F_{c}\left[\dot{x}_{2}(t)\right]+F_{C}\left[\dot{x}_{3}(t)\right]+F_{c}\left[\dot{x}_{1}(t)\right]+F_{S}\left[x_{2}(t)\right]+F_{S}\left[x_{3}(t)\right]+F_{S}\left[x_{1}(t)\right]=u_{B}(t) \\
& m_{3}\left(\ddot{x}_{3}(t)-\ddot{x}_{o}(t)\right)+F_{C}\left[\dot{x}_{3}(t)\right]+F_{c}\left[\dot{x}_{2}(t)\right]+F_{S}\left[x_{3}(t)\right]+F_{S}\left[x_{2}(t)\right]=u_{C}(t)
\end{aligned}
$$

With

$$
\begin{aligned}
& u_{A}(t)=U 1(t)+U 2(t) \\
& u_{B}(t)=U 2(t)+U 3(t) \\
& u_{C}(t)=U 3(t)
\end{aligned}
$$

And

$$
\begin{aligned}
& F_{S}\left[x_{i}(t)\right]=k x_{i}^{3} \\
& F_{c}\left[\dot{x}_{i}(t)\right]=c \dot{x}_{i}{ }^{3}
\end{aligned}
$$

The equations of motion for building can be written in compact form as follows:

$$
M \ddot{x}(t)+C \dot{x}^{3}(t)+K x^{3}=u(t)+M \eta \ddot{x}_{O}(t)
$$

Where

$$
\begin{aligned}
& x(t)=\left[\begin{array}{l}
x_{1}(t) \\
x_{2}(t) \\
x_{3}(t)
\end{array}\right], \quad M=\left[\begin{array}{ccc}
m_{1} & 0 & 0 \\
0 & m_{2} & 0 \\
0 & 0 & m_{3}
\end{array}\right] \quad u(t)=\left[\begin{array}{l}
u_{A}(t) \\
u_{B}(t) \\
u_{C}(t)
\end{array}\right], \quad \eta=\left[\begin{array}{l}
1 \\
1 \\
1
\end{array}\right], \quad C=\left[\begin{array}{ccc}
c_{1}+c_{2} & -c_{2} & 0 \\
-c_{2} & c_{2}+c_{3} & -c_{3} \\
0 & -c_{3} & c_{3}
\end{array}\right] \\
& K=\left[\begin{array}{ccc}
k_{1}+k_{2} & -k_{2} & 0 \\
-k_{2} & k_{2}+k_{3} & -k_{3} \\
0 & -k_{3} & k_{3}
\end{array}\right]
\end{aligned}
$$

Note that $x(t)$ is a three-dimensional vector with $x_{i}(t)$ being the displacement of the ith story; $u(t)$ is a three-dimensional vector consisting of three control forces; $\eta$ is a three-dimensional vector denoting the influence of the earthquake excitation; $M$ is a (3x3) mass matrix and $\mathrm{C}$ and $\mathrm{K}$ are damping and stiffness matrixes respectively.

2.3 Simulation results without controller

The displacements of the three floors are shown in Figure 3, Figure4 and Figure5 respectively. Clearly, the three displacements oscillate around zero with a magnitude of about $0.3 \mathrm{~m}$.

\subsection{Design of A Sliding Mode Controller for a three- story building}

The sliding mode control technique is an attractive control technique which can be used to control linear as well as nonlinear systems. A very important feature of sliding mode controllers is their robustness against parametric uncertainties and un-modeled dynamics such as external disturbances.

In this section, we propose a sliding mode controller to control a three-story building subjected to an earthquake.

Consider the equations of motion for a three-story building subject to a one dimensional earthquake ground acceleration $\ddot{x}_{O}(t)$ and interaction between floors:

$$
\begin{gathered}
m_{1} \ddot{x}_{1}+\left(c_{1}+c_{2}\right) \dot{x}_{1}^{3}+\left(k_{1}+k_{2}\right) x_{1}{ }^{3}-c_{2} \dot{x}_{2}{ }^{3}-k_{2} x_{2}{ }^{3}=u_{A}(t)+m_{1} \ddot{x}_{o}(t) \\
m_{2} \ddot{x}_{2}+\left(c_{2}+c_{3}\right) \dot{x}_{2}{ }^{3}+\left(k_{2}+k_{3}\right) x_{2}{ }^{3}-c_{3} \dot{x}_{3}{ }^{3}-k_{3} x_{3}{ }^{3}-c_{2} \dot{x}_{1}{ }^{3}-k_{2} x_{1}{ }^{3}=u_{B}(t)+m_{2} \ddot{x}_{o}(t) \\
m_{3} \ddot{x}_{3}+c_{3} \dot{x}_{3}{ }^{3}+k_{3}{ }_{3}{ }^{3}-c_{3} \dot{x}_{2}{ }^{3}-k_{3} x_{2}{ }^{3}=m_{3} \ddot{x}_{o}(t)+u_{C}(t)
\end{gathered}
$$

To obtain a state space representation of the above system, we define $z_{i} \quad(i=1, \ldots, 6)$ such that The state space representation of the model of the building is as follows: 


$$
\begin{aligned}
& \dot{z}_{1}=z_{2} \\
& \dot{z}_{2}=\frac{c_{1}+c_{2}}{m_{1}} z_{2}{ }^{3}-\frac{k_{1}+k_{2}}{m_{1}} z_{1}{ }^{3}+\frac{c_{2}}{m_{1}} z_{4}{ }^{3}+\frac{k_{2}}{m_{1}} z_{3}{ }^{3}+\frac{1}{m_{1}} u_{A}(t)+\ddot{x}_{o}(t) \\
& \dot{z}_{3}=z_{4} \\
& \dot{z}_{4}=\frac{c_{2}+c_{3}}{m_{2}} z_{4}{ }^{3}-\frac{k_{2}+k_{3}}{m_{2}} z_{3}{ }^{3}+\frac{c_{2}}{m_{2}} z_{2}{ }^{3}+\frac{k_{2}}{m_{2}} z_{1}{ }^{3}+\frac{c_{3}}{m_{2}} z_{6}{ }^{3}+\frac{k_{3}}{m_{2}} z_{5}{ }^{3}+\frac{1}{m_{2}} u_{B}(t)+\ddot{x}_{o}(t) \\
& \dot{z}_{5}=z_{6} \\
& \dot{z}_{6}=\frac{c_{3}}{m_{3}} z_{6}{ }^{3}-\frac{k_{3}}{m_{3}} z_{5}{ }^{3}+\frac{c_{3}}{m_{3}} z_{4}{ }^{3}+\frac{c_{3}}{m_{3}} z_{3}{ }^{3}+\frac{1}{m_{3}} u_{C}(t)+\ddot{x}_{o}(t)
\end{aligned}
$$

Let the sliding surfaces $S_{1}, S_{2}$ and $S_{3}$ be such that:

$$
\begin{aligned}
& S_{1}=z_{2}+\alpha_{1} z_{1} \\
& S_{2}=z_{4}+\alpha_{2} z_{3}
\end{aligned}
$$

$S_{3}=z_{6}+\alpha_{3} z_{5}$

Where $\alpha_{1}, \alpha_{2}$ and $\alpha_{3}$ are positive scalars.

Also, let $W_{11}, W_{12}, W_{21}, W_{22}, W_{31}, W_{32}, \alpha$ and $\beta$ be positive scalars.

Define the sign function such that:

$$
\operatorname{sgn}\left(S_{i}\right)=\left\{\begin{array}{cc}
1 & S_{i}>0 \\
0 & S_{i}=0 \\
-1 & S_{i}<0
\end{array}\right.
$$

Proposition: The discontinuous static sliding mode controller:

$$
\begin{gathered}
u_{A}(t)=\left(c_{1}+c_{2}\right) z_{2}{ }^{3}+\left(k_{1}+k_{2}\right) z_{1}{ }^{3}-c_{2} z_{4}{ }^{3}-k_{2} z_{3}{ }^{3}-\alpha_{1} m_{1} z_{2}-W_{11} m_{1}\left(z_{2}+\alpha_{1} z_{1}\right) \\
-W_{12} m_{1} \operatorname{sgn}\left(z_{2}+\alpha_{1} z_{1}\right)-m_{1} \frac{z_{2}+\alpha_{1} z_{1}}{\left|z_{2}+\alpha_{1} z_{1}\right|+\alpha e^{-\beta t}} \\
u_{B}(t)=\left(c_{2}+c_{3}\right) z_{4}{ }^{3}+\left(k_{2}+k_{3}\right) z_{3}{ }^{3}-c_{2} z_{2}{ }^{3}-k_{2} z_{1}{ }^{3}-c_{3} z_{6}{ }^{3}-k_{3} z_{5}{ }^{3}-m_{2} \alpha_{2} z_{4} \\
-W_{21} m_{2}\left(z_{4}+\alpha_{2} z_{3}\right)-W_{22} m_{2} \operatorname{sgn}\left(z_{4}+\alpha_{2} z_{3}\right)-m_{2} \frac{z_{4}+\alpha_{2} z_{3}}{\left|z_{4}+\alpha_{2} z_{3}\right|+\alpha e^{-\beta t}} \\
u_{C}(t)=c_{3} z^{3}{ }^{3}+k_{3} z_{5}{ }^{3}-c_{3} z_{4}{ }^{3}-k_{3} z_{3}{ }^{3}-m_{3} \alpha_{3} z_{6}-W_{31} m_{3}\left(z_{6}+\alpha_{3} z_{5}\right) \\
-W_{32} m_{3} \operatorname{sgn}\left(z_{6}+\alpha_{3} z_{5}\right)-m_{3} \frac{{ }^{z}{ }_{6}+\alpha_{3} z_{5}}{\left|z_{6}+\alpha_{3}{ }_{5}\right|+\alpha e^{-\beta t}}
\end{gathered}
$$

When applied to the dynamic model of the three-story building given by equation (13) guarantees the asymptotic convergence of the floor displacements to zero as t tends to infinity. Differentiating equation (14) with respect to time and using (13), (17), (18)and (19) we can write the following:

$$
\dot{S}_{i}=-W_{i 1} S_{i}-W_{i 2} \operatorname{sgn}\left(S_{i}\right)-\frac{S_{i}}{\left|S_{i}\right|+\alpha e^{-\beta t}}+\ddot{x}_{o}(t) \quad \text { for } \quad \mathrm{i}=1,2,3 \text {. }
$$

To prove the existence of sliding mode, we use the following Lyapunov function candidate,

$$
V_{i}=\frac{1}{2} S_{i}^{2} \quad(i=1,2,3)
$$


The time derivative of $V_{i}$ is such that

$$
\begin{aligned}
& \dot{V}_{i}=S_{i} \dot{S}_{i} \\
& =S_{i}\left(-W_{i 1} S_{i}-W_{i 2} \operatorname{sgn}\left(S_{i}\right)-\frac{S_{i}}{\left|S_{i}\right|+\alpha e^{-\beta t}}+\ddot{x}_{o}(t)\right) \\
& =-W_{i 1} S_{i}^{2}-W_{i 2} S_{i} \operatorname{sgn}\left(S_{i}\right)-\frac{S_{i}^{2}}{\left|S_{i}\right|+\alpha e^{-\beta t}}+S_{i} \ddot{x}_{o}(t) \\
& \leq-W_{i 1} S_{i}^{2}-W_{i 2} S_{i} \operatorname{sgn}\left(S_{i}\right)-\frac{S_{i}^{2}}{\left|S_{i}\right|+\alpha e^{-\beta t}}+\left|S_{i}\right| \\
& \leq-W_{i 1} S_{i}^{2}-W_{i 2} S_{i} \operatorname{sgn}\left(S_{i}\right)+\frac{\alpha e^{-\beta t}\left|S_{i}\right|}{\left|S_{i}\right|+\alpha e^{-\beta t}} \\
& \leq-W_{i 1} S_{i}^{2}-W_{i 2} S_{i} \operatorname{sgn}\left(S_{i}\right)+\alpha e^{-\beta t}
\end{aligned}
$$

Taking the limit as $t$ approaches infinity on both sides of the inequality (21) and using Barbalat's lemma, it can be concluded that $\lim _{t \rightarrow \infty} \dot{V}<0$. Therefore, we are guaranteed switching.

The trajectories associated with the discontinuous dynamics (20) exhibit reachability to zero from any given initial condition provided that the constant gains $W_{11}, W_{12}, W_{21}, W_{22}, W_{31}, W_{32}$ are chosen to be large enough strictly positive scalars.

On the sliding surfaces $S_{1}, S_{2}$ and $S_{3}$, we have

$$
\begin{aligned}
& S_{1}=z_{2}+\alpha_{1} z_{1}=0 \\
& S_{2}=z_{4}+\alpha_{2} z_{3}=0 \\
& S_{3}=z_{6}+\alpha_{3} z_{5}=0
\end{aligned}
$$

Therefore, the closed loop dynamics on the sliding surfaces is such:

Hence, we can write,

$$
\begin{aligned}
& z_{2}=-\alpha_{1} z_{1} \\
& z_{4}=-\alpha_{2} z_{3} \\
& z_{6}=-\alpha_{3} z_{5}
\end{aligned}
$$

Clearly (25)-(26) imply that,

$$
\begin{aligned}
& \dot{z}_{1}=-\alpha_{1} z_{1} \\
& \dot{z}_{3}=-\alpha_{2} z_{3} \\
& \dot{z}_{5}=-\alpha_{3} z_{5}
\end{aligned}
$$

$$
\begin{gathered}
z_{1}(t)=e^{-\alpha_{1} t} z_{1}(0) \\
z_{2}(t)=-\alpha_{1} e^{-\alpha_{1} t} z_{1}(0) \\
z_{3}(t)=e^{-\alpha_{2} t} z_{3}(0) \\
z_{4}(t)=-\alpha_{2} e^{-\alpha_{2} t} z_{3}(0) \\
z_{5}(t)=e^{-\alpha_{3} t} z_{5}(0) \\
z_{6}(t)=-\alpha_{3} e^{-\alpha_{3} t} z_{5}(0)
\end{gathered}
$$


Therefore, it can be concluded from (27) that $z_{i}(t) \quad(\mathrm{i}=1, \ldots, 6)$ converges to zero as $\mathrm{t}$ tends to infinity.

Hence, the controller (17), (18),(19) when applied to the dynamic model of the building given by equation (13) guarantees the asymptotic convergence of the floor displacements to zero as $t$ tends to infinity.

\subsection{Simulation results by using controller}

The displacements of the three floors are shown in Figure 6, Figure7 and Figure8 respectively. Clearly, the displacements have been reduced.

\section{Conclusion}

The figures $6,7,8$ clearly show the controllers have dramatically reduced the amplitudes of the oscillations of the three floors. It is observed that the controllers reduce the peak responses of the displacements of every floor. The first floor has a reduction from $0.25 \mathrm{~m}$ to $12 \times 10^{-5} \mathrm{~m}$, the second floor has a reduction from $0.3 \mathrm{~m}$ to $12 \times 10^{-5} \mathrm{~m}$ and the third floor has a reduction from $0.35 \mathrm{~m}$ to $12 \times 10^{-5} \mathrm{~m}$.

\section{References}

Agrawal, A. K. Yang, J. N. and Schmitendorf, W. E. (1998). Hybrid Control of Buildings Using Nonlinear Polynomial Output Feedback. The American Control Conference (Philadelphia, Pennsylvania), 4, 2554-2558.

Agrawal, A. K. Yang, J. N. (1995). Nonlinear Output Feedback Control for Seismic-Excited Linear Structures. The American control conference, 3, 1921-1925.

Agrawal, A. K. and Yang, J. N. (1997). Effect of Time-Delay on Multi Degree of Freedom Actively Controlled Civil Engineering Structures. The American control conference, 1, 21-25.

Lu, L. Y. Lin, G. L. Kuo, T. C. (2008). Stiffness Controllable Isolation System for Near-Fault Seismic Isolation. Engineering Structures, 30(3), 747-765.

Yang, J. N. Agrawal, A. K. (2002). Semi-Active Hybrid Control Systems For Non-Linear Buildings Against Near-Field Earthquakes. Engineering Structures, 24(3), 271-280.

Earthquake Hazard program http://earthquake.usgs.gov.

The National Geophysical Data Center http://www.ngdc.noaa.gov.

Mid-America Earthquake Center (MAE). http://mae.ce.uiuc.edu.

The American Association for Wind Engineering http://www.aawe.org.

Fujino, Y. Soong, T. T. and Spencer, B. F. (1996). Structural Control: Basic Concepts and Applications. Proc. ASCE Structures Congress XIV. (Chicago, Illinois), 1277-1278.

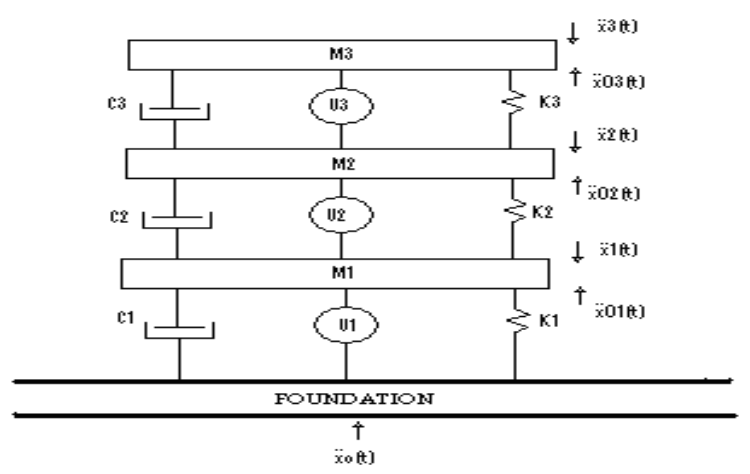

Figure 1. A Three- Story Base-Isolated Building Model 


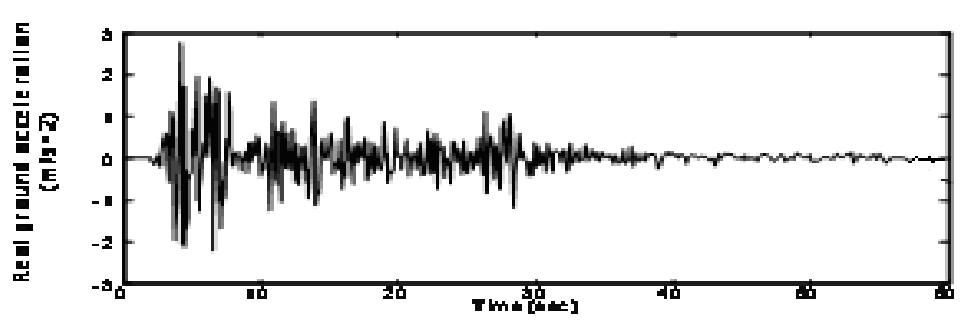

Figure 2. $\ddot{x}_{o}(t)$ Ground acceleration (Real)

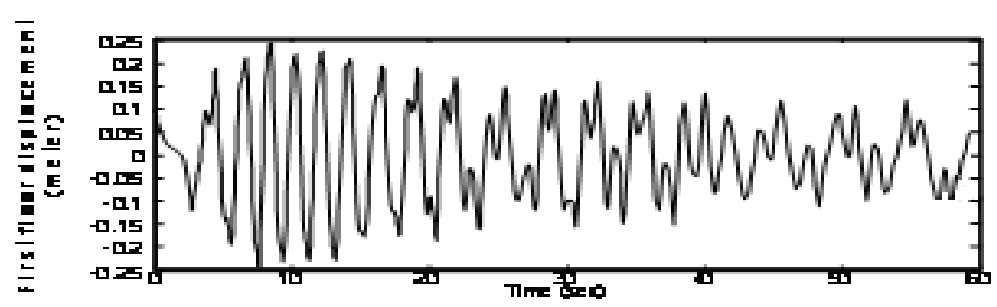

Figure 3. $x_{1}(t)$ First floor displacement

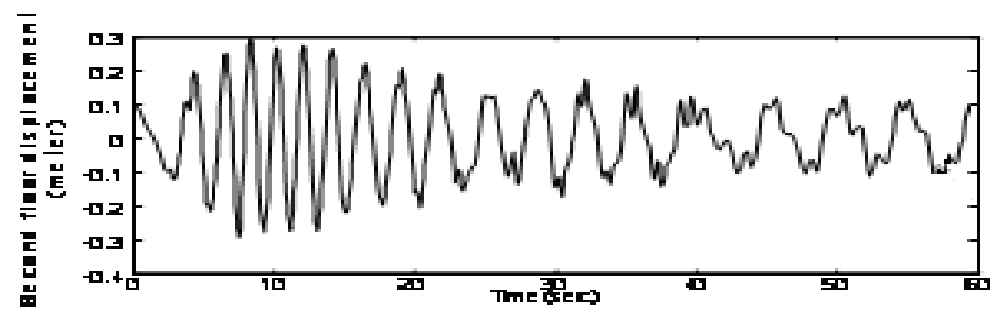

Figure 4. $x_{2}(t)$ Second floor displacement

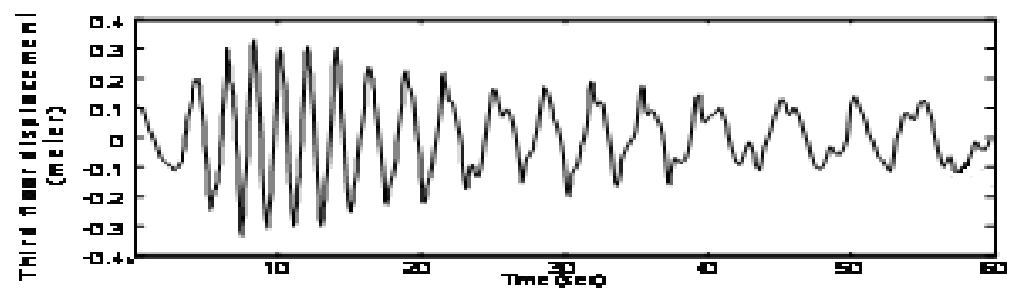

Figure 5. $x_{3}(t)$ Third floor displacement

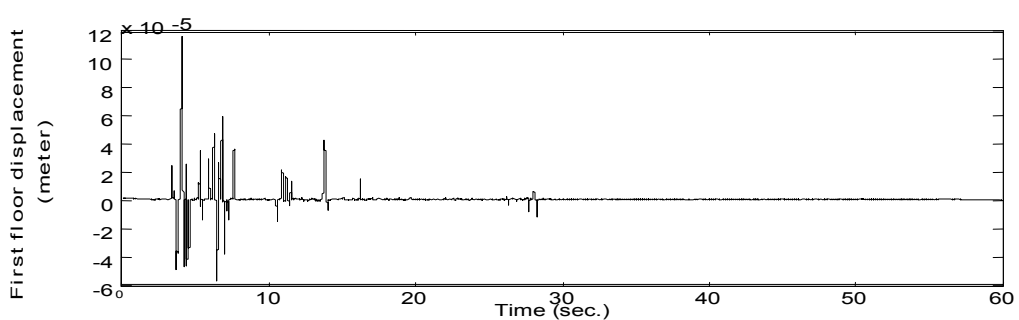

Figure 6. First floor displacement by using controller 


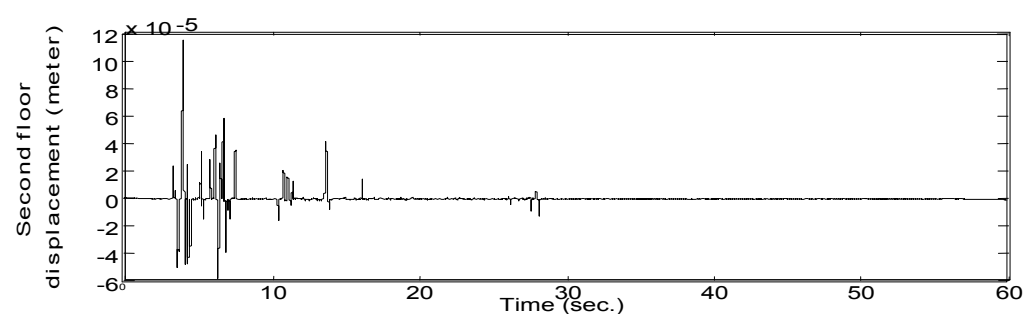

Figure 7. Second floor displacement by using controller

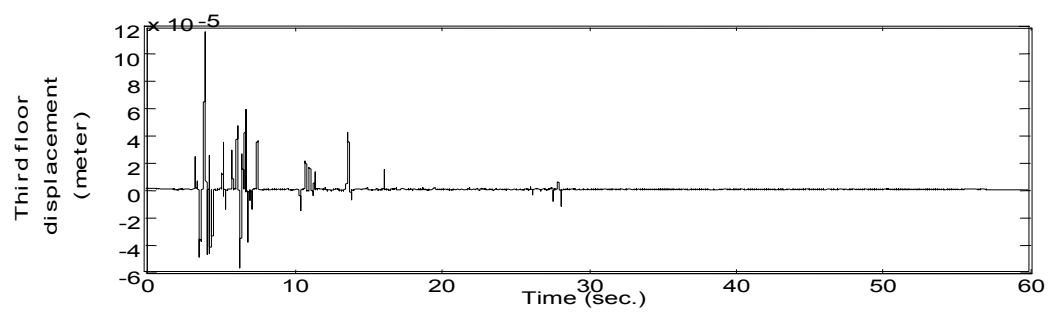

Figure 8. Third floor displacement by using controller

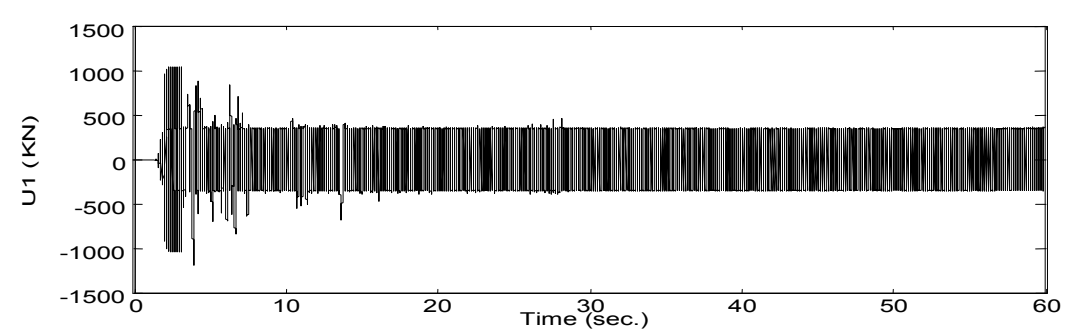

Figure 9. First floor controller

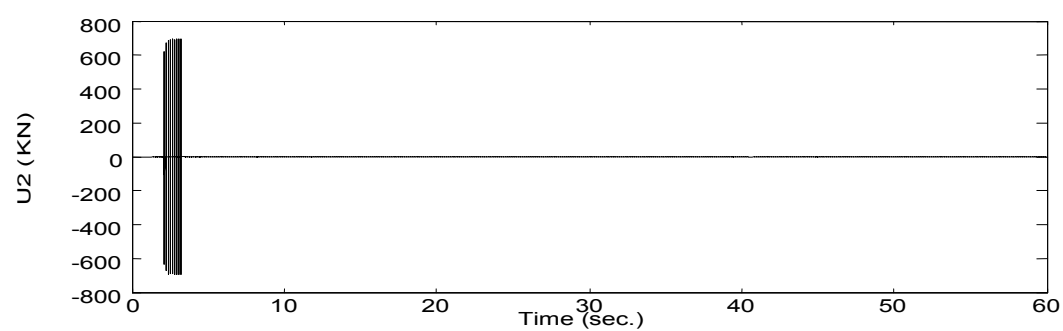

Figure 10. Second floor controller

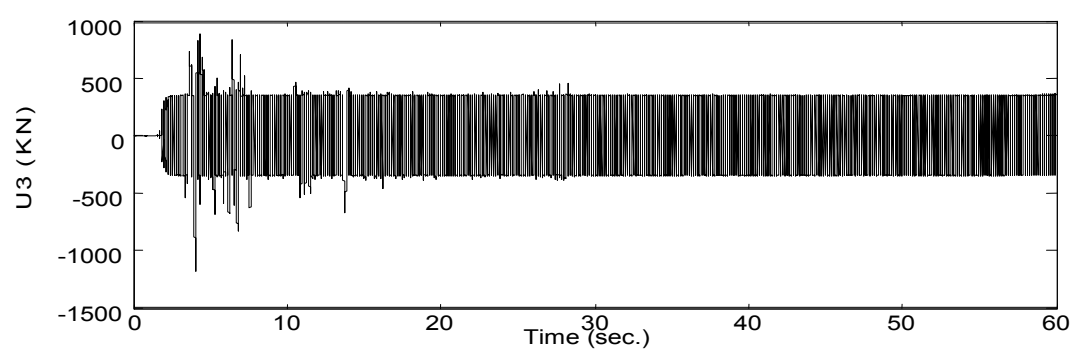

Figure 11. Third floor controller 